\title{
Application of Blockchain Technology in the Field of Medical Devices
}

\author{
Qian $\operatorname{Min}^{1, *}$, Wang Shuting ${ }^{2, a}$ \\ ${ }^{1}$ Xi' an university of science and technology, School of Management, Xi'an, Shanxi province \\ ${ }^{2} \mathrm{Xi}$ 'an university of science and technology, School of Management, Xi'an, Shanxi province
}

\begin{abstract}
In recent years, Blockchain technology has been mentioned for many times in the fields of finance, Internet and energy, and it is a distributed data ledger that is decentralized, authentic, reliable, traceable open and transparent. Blockchain is constantly figuring out how to integrate with other industries. This paper analyzes the current situation in the field of medical devices of China by combing the Annual Report on Medical Device Adverse Event Monitoring from 2013-2019, and using the compound annual average growth rate. The analysis found that this field has pain points such as lack of third-party regulation and difficulty in traceability, and then it proposed the specific application of blockchain technology in the field of medical devices to solve the problems in this field.
\end{abstract}

\section{Introduction}

Blockchain has been mentioned for many times in recent years in the financial field, Internet field and energy field, mainly because of its unique features of decentralization, full traceability, traceability, openness and transparency. These features guarantee the absolute "honesty" and "transparency" of the blockchain, making it trusted by most people to a great extent.

Blockchain is well known mainly because of Bitcoin. In 2018, Satoshi Nakamoto first proposed the design concept of decentralized cryptocurrency-Bitcoin [1]. Bitcoin is one of the most successful application scenarios of blockchain technology. The official birth of Bitcoin was in 2009, when the Bitcoin system began to operate on the Internet; from 2010 to 2015, Bitcoin gradually gained media attention and entered the public view, and the US e-commerce giant eBay accepted Bitcoin payments; From 2016 to 2020, Bitcoin has received great attention in various countries, while different countries have different attitudes towards Bitcoin. For instance, the central bank has issued an announcement saying that virtual currency transactions are prohibited. Behind this, the more important thing is to witness the application and development of blockchain. With the prevalence of virtual currency, people gradually believe that blockchain can build a reliable trust value chain in a scenario where multiple parties participate. In 2013, programmer Vitalik Buterin was inspired by Bitcoin and proposed Ethereum ${ }^{[2]}$. It is based on blockchain technology and is more like a platform on which anyone can use blockchain technology to construct decentralization. In this application, Solidity is used to write smart contracts that interact with the blockchain ${ }^{[3]}$.At present, Ethereum is gradually being used in more industries due to its simplicity and universality.

This article is mainly to build a scenario application of medical devices or rings based on blockchain technology. With the development of science and technology, Chinese medical equipment has also made rapid progress, and more and more medical equipment begin to be customized, especially after the introduction of $3 \mathrm{D}$ printing technology. However, what follows is the issue of quality supervision. Medical device companies on the market have different technical levels, leading to uneven product quality in the market, and the medical accidents caused by quality problems of medical devices are gradually increasing.

Since 2013, in order to comprehensively reflect and monitor the adverse events of medical devices in China, the National Center for Adverse Drug Reaction Testing compiled the Annual Report on National Medical Device Adverse Event Monitoring, whose data collection mainly relies on the registration reports of different role parties in the system, and the data has certain limitations and incompleteness. The number of cases recorded in the annual report increased from 366 in 2003 to 396345 in 2019 , which is only on record, so it is conceivable that there are definitely a lot of accidents caused by medical devices. In addition to strengthening government supervision, we should use modern means to supervise the whole process of medical devices. On the one hand, it can make the production, manufacturing, transportation and use process traceable and transparent, and subjectively increase the responsibility of each link; on the other hand, if an accident occurs, it can better identify the responsible party to facilitate handling. The 
blockchain technology is combined with the medical device scene.

\section{Status of medical device adverse events}

\subsection{Adverse event analysis}

The analysis of medical device adverse events mainly uses visual tools for descriptive analysis, using statistical data from the "Annual report on Medical device adverse event Monitoring" published on the official website of the State Drug Administration from 2013 to 2019. Descriptive analysis of trends using compound annual average growth rates [4] was calculated as follows.

$$
C A G R\left(t_{0}, t_{n}\right)=\sqrt[t_{n}-t_{0}]{\left(\frac{V\left(t_{n}\right)}{V\left(t_{0}\right)}\right)}-1
$$

\subsubsection{Number of medical device adverse events reported nationwide}

From 2003 to 2019, the number of reports of medical device adverse events in the country continued to increase, from 366 in 2003 to 396,345 in 2019, with a compound annual growth rate of $54.76 \%$, as shown in Figure 1. Since the country did not formally write the report from 2003 to 2010 , the base figure is relatively small, and the fluctuations in the first and last years of growth are more obvious. In comparison, the growth was relatively stable from 2010 to 2019 , with a compound annual growth rate of $23.34 \%$. From 52 cases per million populations in 2010 to 297 cases in 2019 , the compound annual growth rate in 9 years was $21.36 \%$.

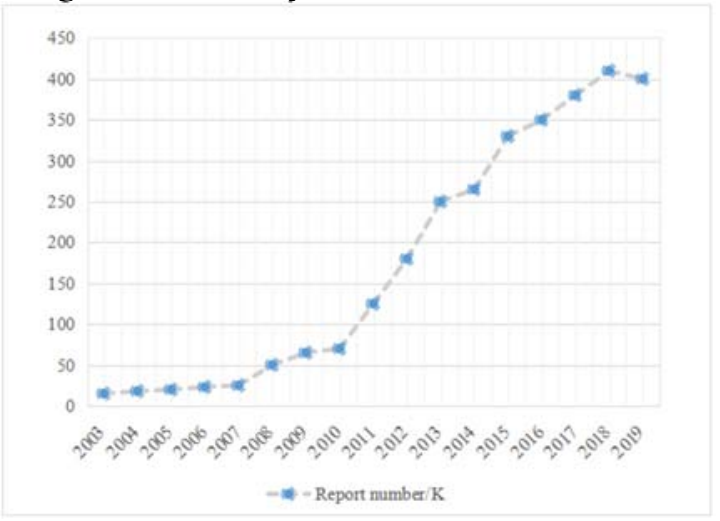

Fig.1. Number of medical device adverse events reported nationwide

\subsubsection{Source for reporting adverse events of medical devices}

The main source of information about medical device adverse events is the "National Medical Device Adverse Events Monitoring System". The number of registered grassroots users of the system increased from 148,585 in 2013 to 318,986 in 2019 , with a compound annual growth rate of $11.5 \%$ over the past seven years. The grassroots users registered in 2019 are operating companies, using companies, and production companies, and their proportions are $55.89 \%, 37.94 \%$, and $6.16 \%$. In terms of the changes in composition from 2013 to 2019, the proportion of production companies is relatively stable, while the operating companies and using companies have changed, as shown in Figure 2.

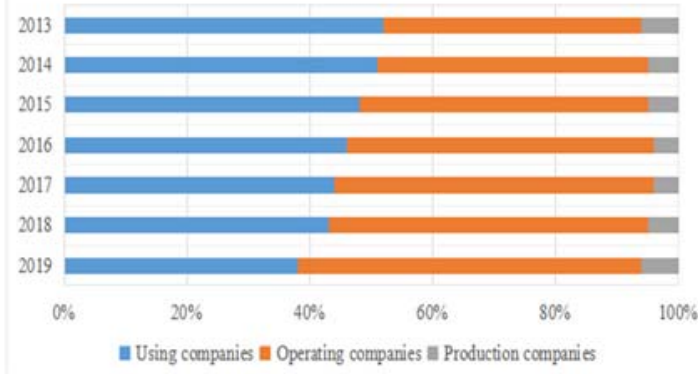

Fig.2. 2013-2019 registered user profile composition ratio

From the perspective of the composition of the sources of medical device adverse event reports in 2019, the highest proportion of incidents reported by enterprises was $90.27 \%$, operating enterprises were $7.53 \%$, and manufacturing enterprises were $2.17 \%$. From 2013 to 2019 , the source composition of the report did not change much. Judging from the quantitative changes in the past seven years, the number of companies reporting has continued to grow in the past seven years, with a compound annual growth rate of $51.7 \%$, as shown in Figure 3.

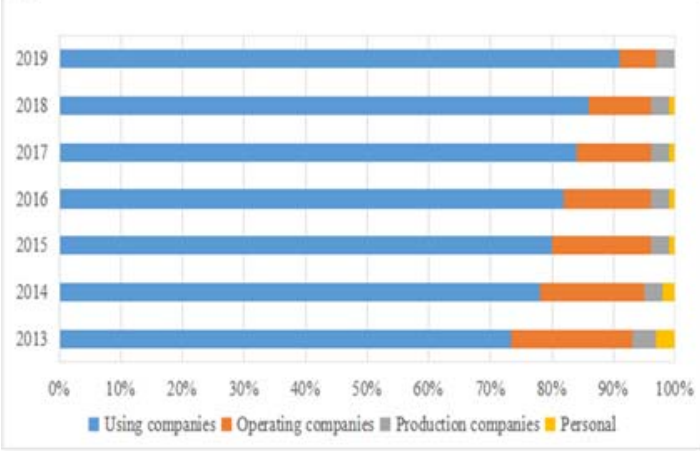

Fig.3. 2013-2019 Source Composition Ratio

It can be seen from the above report that different types of medical device institutions have completely different attitudes towards registration and declaration, which has certain relevant benefits for them. The system must be manually registered, filed, and declared. Many companies still have concerns about reporting adverse events, believing that it will have a great impact on corporate reputation. Therefore, from the perspective of obtaining data, the data is largely incomplete and lacks authenticity, which poses a great challenge and difficulty to the regulatory testing in the development of Chinese medical device industry. 


\section{Blockchain in Medical Devices}

\subsection{Blockchain Features}

The essence of the blockchain is a different database from the past, composed of different blocks. Different blocks can be connected, recorded and maintained together, and they become endorsements of each other and exist in the same ledger. Once new information is added, the ledger is updated immediately, and neighboring nodes will immediately record it, and it cannot be tampered with. If the Bitcoin system is called the blockchain 1.0 era, the emergence of Ethereum gave birth to the blockchain 2.0 era, from data to value realization. Ethereum has a built-in complete programming language, which can be used to build various applications. The emergence of Ethereum smart contracts realizes automatic programming and automatic operation on the chain, creating new commercial value for the application of blockchain in various industries. Its characteristics are reflected in the following 4 aspects:

1) Information Sharing

The core of blockchain itself is decentralized data storage technology, where the data between different nodes can be shared with each other to reach a consensus mechanism, which is transparent and reliable for all nodes' information on the chain.

2) Authentic and Secure Information

Blockchain is based on the principle of cryptography for data protection, which makes it impossible to modify the data as long as $51 \%$ of the nodes are not in control so that the balance is out of balance, which allows the information itself to be protected from human tampering with the data.

3) Information traceability

The information is completely traceable when using blockchain technology. The blockchain is a chain structure, and there will be node heads and node tails on this chain. Each node consists of a block header and a block body, and there is also the Timestamp composition. The block header records the hash value of the previous node, and the block body stores data. If the information of the previous node changes, the hash value will change, which will be different from the hash value stored in the next block header, and you can find the difference in information. Any data can be traced along the chain to the point of origin.

4) Smart Contract

A smart contract is a piece of code on the blockchain that automatically executes a contract when conditions are met,without the authorization of a third party or intermediary to run it. As a result, smart codes can be used to develop decentralized applications with commercial value.

\subsection{Blockchain Type}

Blockchains are divided into three types: public, private, and alliance chains ${ }^{[5]}$. The public chain is developed for all people and does not require access permission, and anyone can participate in it, and virtual currencies such as Bitcoin are based on the public chain; the alliance chain is managed by several organizations, and the nodes are mutually recognized and require access permission, and the alliance chain can be mostly used in blockchain application scenarios; the private chain also requires access permission and is completely closed, and is often controlled by Private chains also require access licenses and are completely closed, often in the hands of an individual or an organization.

\subsection{Blockchain in Medical Devices}

The current medical device market in China has adverse events. The existing adverse event reporting system has obvious shortcomings. On the one hand, it relies on manual reporting, which is difficult to report, and the registration system covers incomplete populations. Individual registration of affected patients accounts for the least amount. Negligible; on the other hand, it is more difficult to pursue responsibility if there is a problem with the device.

Introducing blockchain into the field of medical devices, as a technology that uses a decentralized consensus mechanism to maintain a non-tamperable ledger database, blockchain can realize all aspects from the initial diagnosis of patients to the design, production, logistics, traceability and disclosure of the whole process of final use. Most of the information in the blockchain technology is recorded with a hash code that does not reveal any user information. In the whole process, the privacy and due rights of consumers are protected. For the entire industry, it can reduce the time for clearing and settlement, reduce costs, and reduce vicious competition among enterprises, and if any node on the chain has a problem, it can be traced back to upstream and downstream nodes.

\subsubsection{Business Process}

When patients go to the hospital, they first enter their identity information and medical history in the system; according to their medical condition, they use or customize the medical devices they need in the process; if they only use the medical devices, they enter the corresponding barcode of the medical devices; if they need customized medical devices, they first upload the patient's needs to the database; the products that meet their needs start to be made; the products are shipped; the medical devices are installation; product after-sales. The business process is detailed in Figure 4. 


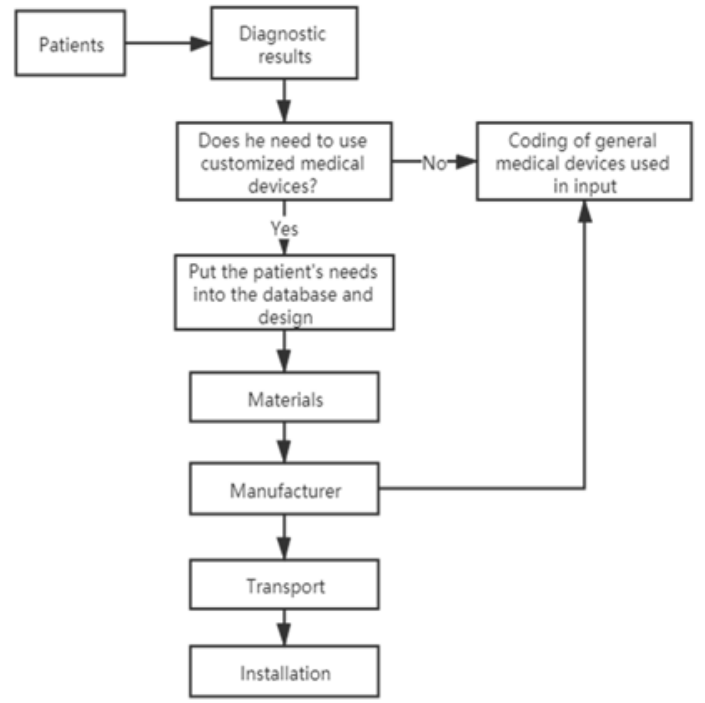

Fig.4. Business Flow

The entire process can be accessed by the patient through the APP. The user does not log on the chain, and only the underlying data is realized by the blockchain. Moreover, because the blockchain has the characteristics of data that cannot be tampered with, the designed business process has the characteristics of information disclosure, data transparency and simple operation. The entire process information is traceable throughout, and patients can see the production data of the devices they use through the APP. If any adverse event occurs, it provides great convenience when investigating medical devices.

\subsubsection{The way to achieve}

Since medical care is a public utility, the government can shut down and take the lead in building an alliance chain, allowing five types of nodes to enter: hospitals or other medical institutions, manufacturers or equipment, consumables or equipment suppliers, government regulatory agencies, logistics and warehouses.

Using hospitals or other medical institutions as the chain end, if patients do not need customized medical devices, the devices used will be connected directly from the warehouse; if customized medical devices are needed, the patient visit information will be transmitted and the manufacturer will manufacture according to the requirements and generate the order number.

The manufacturer first sets the parameters of the equipment according to the patient's needs, then enters the raw materials or equipment codes used, and records them directly in the order number when archiving.

After purchasing the raw materials, the production manufacturer will proceed directly to the production, download the information of the instrument parameters through the order number, and update the information on the chain after the production is completed.

Government regulators directly open the order information, review the compliance of the raw material, as well as approve whether the production manufacturer is qualified to produce, whether it meets the national medical standards and production and production standards, and whether there are any violations, and after the audit is completed, the chain order is updated.

In the links in the logistics and warehousing, confirm that the product is approved, and determine the delivery time of the product. The product status needs to be updated when it is delivered.

As shown in Figure 6, the structure of the medical device traceability federated chain is as follows, and its inter-block connections are formed as a data chain in the order of time stamps, and the data on the chain have natural correlation.

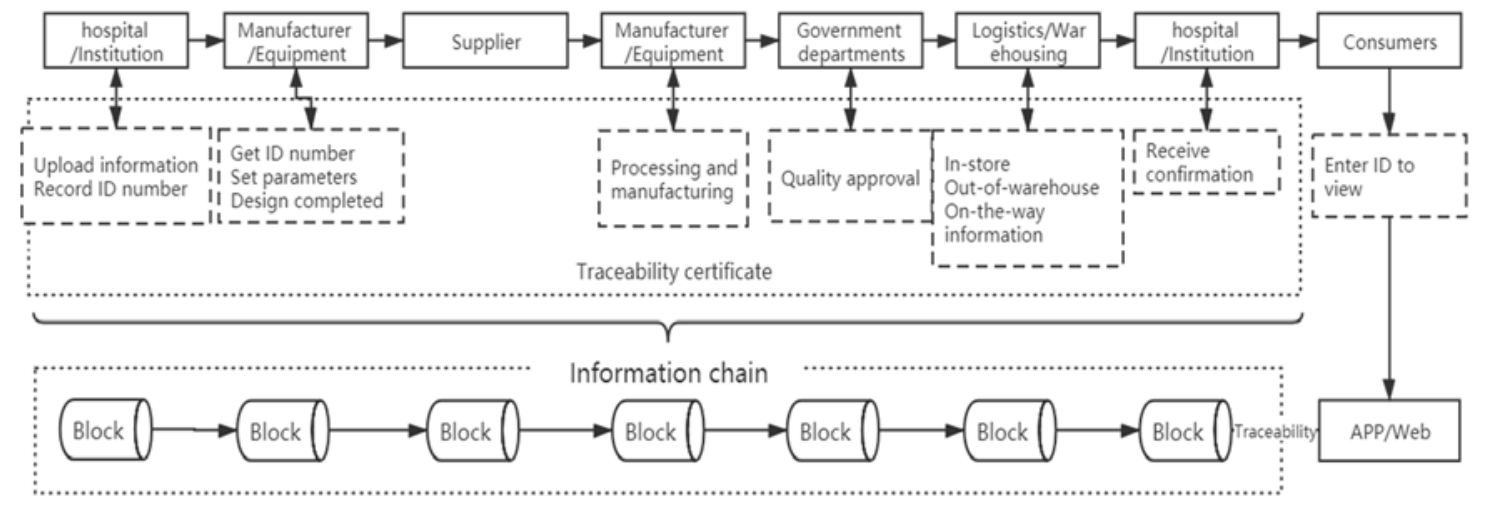

Fig.5. Medical Device Traceability Alliance Chain

\subsubsection{Block data operation}

Firstly, each transaction must start with the generated order ID, and the content of the next line is the time when the information is updated, and the update time is automatically recorded by the system. Within the same transaction, UTCTime is automatically generated. Each piece of information can only update one new piece of information at a time. The information marked in green is the updated information, and each piece of information updated afterwards is continuous after the previous piece of information, That is, except for the last message that was newly edited and the time was automatically recorded by the system, the rest of the information was previously fixed. As shown in Figure 7, except for the 
last message and UTCTime, all other messages should be colored.

\begin{tabular}{|c|c|}
\hline BLOCK: 1 & BLOCK: 2 \\
\hline LastBlock: 〈gensisBloack> & LastBlock: \\
\hline UTCTime: 20201201110000 & $2 e 0 e 95285 f 08 a 07 d e a 17 e 7 e e 111 \mathrm{~b} 21 \mathrm{c} 8$ \\
\hline Information Included: 2 & UTCTime: 20201202120040 \\
\hline Merkel root: 〈hash〉 & Information Included: $\mathbf{2}$ \\
\hline \multirow{3}{*}{$\begin{array}{l}\text { Order ID: } 2020150401 \text { PVG001 } \\
\text { UTCTime: } 20201201112054 \\
\text { Model ID: 〈hash> }\end{array}$} & \\
\hline & Order ID: 2020 SS0401PVG001 \\
\hline & UTCTime: 20201202122031 \\
\hline \multirow{5}{*}{$\begin{array}{l}\text { Order ID: } 2020 \text { SH0401HKU001 } \\
\text { UTCTime: } 20201201113051 \\
\text { Model ID: ‘hash〉 } \\
\text { Supplier: XILU01MTH089 }\end{array}$} & $\begin{array}{l}\text { Model ID: ‘hash> time: Zute> } \\
\text { Supplier: CHNS09PHY81 Pattem: 〈hash> }\end{array}$ \\
\hline & \\
\hline & Order ID: 2020 SH0401HKU00I \\
\hline & UTCTime: 20201202123051 \\
\hline & Model ID: 〈hash〉 time: 〈ute \\
\hline & Supplier: XILU01MTH089 Pattem: 〈hash> \\
\hline
\end{tabular}

Fig.6. Block Structure

When updating information, first use the order number to trace back, and then access the information with the last time stamp to verify whether the information record before this link is sufficient. After passing the verification, the UTC time of this message is appended to the last message, so far all the messages become colored. Add two more new messages. Since there is no need to mine, the block header is much simpler than Bitcoin's blockchain, which is simply the hash value of the entire block of the previous block ${ }^{[6]}$.

At present, in order to realize the application of blockchain technology in the medical device industry, it is necessary to combine the Internet of things, intelligent sensing, big data acquisition and other technologies. For example, matching medical device products with identification marks such as traceable source code and electronic labels, which can prevent problems in the circulation process.

\section{Conclusion and Application}

Generally, the blockchain technology can be applied in the field of medical devices. Firstly, from the source of each link, the quality and safety of medical devices will be greatly guaranteed. Because of the traceability of the blockchain, every link from the order to the use of the whole process receives accurate and reliable "supervision", and the responsibility is clear. Secondly, the smart contract of the blockchain makes government supervision more efficient and convenient. It only needs to set relevant government departments into the nodes of the alliance chain to supervise all production and operation processes and make supervision more standardized. Thirdly, due to the immutability of the blockchain, the data on the chain is all true and reliable, making the market more open, transparent and standardized.

The application of blockchain technology in various scenarios is still in the exploratory stage, and the technology itself is still in continuous development. At present, it is widely combined with the financial field and energy field, but there are also some problems. How to combine blockchain technology with traditional industries needs to be explored constantly. Looking to the future, the $5 \mathrm{G}$ era has arrived, and the interconnection of everything has gradually become the development trend of various industries and the direction of upgrading and optimization. It is believed that the development of blockchain in combination with other technologies in various industries will be more comprehensive and effective.

\section{References}

1. NAKAMOTOS.

Bitcoin: apeer-to-peerelectroniccash system. (2019). https://bitcoin.org/bitcoin.pdf.

2. BUTEINV.Anext-generationsmartcontractanddecent ralizedapplicationplatform.(2018)https://github.com/ ethereum/wiki/wiki/White-Paper.

3. SZABON. The Idea of Smart Contracts. (2018). http://szabo.best.vwh.net/smart contracts idea.html.

4. Si Zhu.Situation analysis on the adverse events monitoring of medical device in China. (Chinese Health Resources,2019).

5. Jinyong Shan, Sheng Gao..Advances in Blockchain Theory Research.(Journal of Cryptologic Research, 2018)

6. KATZJLINDELLY. Introduction to modern cryptography $[\mathrm{M}] .2$ nded. BocaRaton, USA: ChapmanandHall/CRC, 2008. 